Meta

Journal des traducteurs

Translators' Journal

\title{
On ne naît pas interprète, mais cela aide!
}

\section{Georges L. Bastin}

Volume 33, numéro 4, décembre 1988

Symposium AILA 1987, Sydney

URI : https://id.erudit.org/iderudit/001985ar

DOI : https://doi.org/10.7202/001985ar

Aller au sommaire du numéro

Éditeur(s)

Les Presses de l'Université de Montréal

ISSN

0026-0452 (imprimé)

1492-1421 (numérique)

Découvrir la revue

Citer cette note

Bastin, G. L. (1988). On ne naît pas interprète, mais cela aide! Meta, 33(4),

582-586. https://doi.org/10.7202/001985ar d'utilisation que vous pouvez consulter en ligne.

https://apropos.erudit.org/fr/usagers/politique-dutilisation/ 


\section{ON NE NAITT PAS INTERPRÈTE, MAIS CELA AIDE!}

... il est faux de prétendre que l'on naît traducteur comme on naît poète, et que tout effort pour former des traducteurs est vain. $\mathrm{J}$ 'espère que ceux qui sont convaincus du contraire ont la décence de se tenir loin des écoles de traduction, car l'imposture serait assez grossière. (Jean Delisle)

La plupart des professionnels de l'interprétation, formés dans l'une ou l'autre école ou «sur le tas ", conviendront que leurs aptitudes ne leur sont pas tombées du ciel mais qu'au contraire elles représentent le fruit de longues années d'efforts. Il leur serait néanmoins difficile de nier que les chances d'aboutir soient égales pour tous. Quant à nous, enseignants, nous sommes les témoins directs de l'un et de l'autre. C'est à nous en effet qu'incombe la tâche de développer ce "minimum de savoir-faire pour exercer convenablement ce métier" ". Et Jean Delisle de poursuivre : "Par sayoir-faire, j'entends l'aptitude à interpréter le sens d'un texte pour être en mesure de le restituer clairement... Nous nous trouvons, si je puis dire, aux premières loges de la transformation de l'étudiant-de-la-rue en futur interprète. Qui plus est, nous devons être les artisans de cette mutation.

Qu'est-ce qui nous permet d'affirmer que nous serons en mesure d'aider la chrysalide à se transformer en papillon? Toutes les larves deviendront-elles lépidoptères? Comment faire en sorte qu'elles y parviennent? Autant de questions auxquelles nous tenterons de répondre, nous fondant sur une expérience de cinq années d'enseignement de l'initiation à l'interprétation à l'École de langues modernes de l'Université centrale du Venezuela 2 .

\section{TRADUCTION ET COMMUNICATION}

S'inspirant des recherches de Danica Seleskovitch et de son équipe, Daniel Gile écrit : "... l'activité traduisante n'est plus perçue comme un transcodage linguistique mais comme une opération en deux temps :

- extraction d'un "message" à partir de l'énoncé original

- production d'un deuxième énoncé en fonction des aptitudes et habitudes linguistiques du traducteur ${ }^{3}$ "

Cette perception n'est peut-être pas neuve mais elle revêt un caractère de plus en plus actuel. Elle est surtout réelle et professionnelle. Il y a longtemps que les interprètes professionnels qui se préoccupent d'observer et d'étudier notre "savoir-faire "font $f$, dans un premier temps, de toute considération de transfert purement linguistique. Dans ce premier temps, qui intéresse au premier chef les enseignants, l'acti- vité traduisante n'est autre chose qu'une opération de communication. Non seulement la traduction (en tant qu'activité intellectuelle) fait partie intégrante du processus de la communication mais elle est et ne pourrait être autre que communication.

Si donc la traduction est communication, la réponse à la troisième question doit être la suivante : "Apprendre à nos futurs interprètes à communiquer ". D'aucuns ne verront là qu'une vision simpliste du problème car, diront-ils, depuis notre plus tendre enfance nous communiquons - vrai! - et tous, nous savons communiquer - faux! Les relations humaines, dans quelque domaine que ce soit, se caractérisent quotidiennement par une incapacité généralisée d'écoute et d'expression. Un manque patent de communication efficace. Nos étudiants n'y font pas exception.

Les facteurs déterminants de l'efficacité de la communication, tels ceux définis par David $\mathbf{K}$. Berlo ${ }^{5}$, entre autres, peuvent contribuer à jeter une lumière neuve sur les ingrédients de nos recettes d'initiation à l'interprétation visant à faire de l'étudiant-de-la-rue un interprète potentiel. Berlo mentionne quatre facteurs qui coïncident avec ceux énoncés par la plupart des auteurs : Aptitudes communicatives - Attitudes - Niveau de connaissances - Système socioculturel.

Par aptitudes communicatives, Berlo entend l'écoute, l'expression et la pensée. Écoute, soit décodage; expression, soit encodage. Jusque-là, rien de neuf : deux aptitudes fondamentales qu'il convient de développer chez nos étudiants par divers exercices. Mais, la pensée... un troisième temps à ajouter à ceux décrits par $\mathrm{D}$. Gile. Un temps intermédiaire que D. Seleskovitch a amplement mis en évidence dans son ouvrage Langage, langues et mémoire ${ }^{6}$ et qui nous oblige irrémédiablement à envisager le processus mental de l'interprétation sous le jour inéluctable de notre réalité professionnelle. Car, en fin de compte, s'il est possible que certains interprètes se sentent frustrés " par l'aspect travail de perroquet de l'interprétation de conférence ${ }^{7}$ ", je doute qu'ils fassent la majorité. Qu'il s'agisse d'appréhender un message ou de l'exprimer, l'homme requiert une certaine capacité de pensée, une aptitude à associer des symboles mentaux, des images directement liées à son expérience socio-intellectuelle. Cette faculté, sans laquelle il ne peut y avoir de transfert de contenus ne tient en rien du psittacisme. Elle se manifeste généralement de manière inconsciente dans un acte de communication normale entre deux interlocuteurs. Néanmoins, l'interprète n'étant pas un interlocuteur puisque les contenus ne lui sont pas destinés, * il est celui qui écoute aux portes un échange entre initiés ${ }^{8}$, il appartient aux écoles d'interprètes d'insuffler ou de développer cette compétence chez leurs étudiants. Sans pensée, pas de compréhension ni d'expression.

Le niveau de connaissances et la conscience des systèmes socioculturels déterminent également. nul n'en peut douter, le degré d'efficacité de toute communication. On ne peut, en effet, communiquer 
ce qu'on ignore. De même, l'ignorance des réalités socioculturelles auxquelles appartiennent tant l'émetteur que le récepteur fait de la communication un dialogue de sourds. Cependant nous ne pouvons pas perdre de vue que l'interprète en situation ne partage que rarement le niveau de connaissances et le système socioculturel des interlocuteurs. De nouveau, les écoles ont là un rôle de premier plan à jouer.

L'efficacité de la communication est également fonction, toujours selon Berlo, d'un autre facteur, les attitudes, qui intéresse particulièrement les futurs interprètes. Combien de fois les étudiants ne se plaignent-ils pas d'un professeur lisant son cours, pourtant excellent, le nez enfoui dans ses notes? Le professeur d'un étudiant exposant un sujet sans exhiber le moindre intérêt? L'interlocuteur de l'autre perdu dans ses rêveries? Il s'agit bien là d'attitudes : envers le destinataire, envers le sujet traité, envers l'émetteur. Trois types d'attitudes propres à la plupart des situations de communication. Il en est un autre fondamental pour tout communicateur et en particulier pour l'interprète : l'attitude envers soimême. Beaucoup de nos étudiants - trop en tout cas - souffrent de ne pas avoir conscience du potentiel qui dort en eux. C'est à l'enseignant de l'éveiller. Nombreux sont ceux qui cessent d'être eux-mêmes lorsqu'ils entament un exercice d'interprétation; ils ont le trac, ils "perdent les pédales" parce qu'ils nnanquent de confiance en eux. Une défaillance qu'ils surmonteront difficilement seuls, devant un miroir, par exemple, comme certains le recommandent. Le cours d'initiation à l'interprétation ne peut manquer l'objectif capital de donner à l'étudiant l'assurance qui lui permettra de communiquer efficacement

L'intérêt de développer parmi nos étudiants les aptitudes fondamentales d'écoute et d'expression ainsi que la prise de conscience de l'importance à accorder au niveau de connaissances, aux systèmes socioculturels et aux attitudes ne doit en aucun cas occulter le caractère propre d'une " étape intermédiaire pendant laquelle le signifiant disparaît alors qu'interviennent des mécanismes cérébraux non linguistiques $^{9} \%$

\section{TRADUCTION ET ABSTRACTION}

Dans une communication au colloque $\& \mathbf{L a}$ traductologie " de Mons, je mentionnais parmi les obstacles à l'enseignement universitaire de la traduction en Amérique latine « un asservissement total à la forme, même malheureuse, aux dépens de "vouloirdire " limitant ainsi la capacité d'abstraction, condition sine qua non de l'exécution d'une traduction digne de ce nom ${ }^{10}$ ". Par capacité d'abstraction, entendons la faculté d'" extraire le sens ... saisi sous une forme déverbalisée, c'est-à-dire libérée des signes

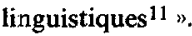

Les exercices unilingues d'extraction des notions clés (tels ceux proposés pour la traduction par Jean Delisle dans son "Analyse du discours comme méthode de traduction ") appliqués à l'oral avec des étudiants d'interprétation en début d'apprentissage révèlent immédiatement les difficultés d'affranchissement de la forme et, surtout, d'abstraction des idées entendues. S'il est vrai que, souvent, la plupart des unités de pensée correspondent à des unités de langage $^{12}$, il est indispensable d'habituer le futur interprète à dissocier les unités de pensée de la langue, à les ordonner, à les mémoriser sans qu'intervienne nécessairement une formulation linguistique. Après audition d'un fragment de texte (de préférence anecdotique ou argumentatif) dont l'étudiant est appelé à extraire les idées clés (sans note), il convient de repasser le film ou le fil du sens, de signaler à l'étudiant le cheminement du vouloir-dire, lui proposer (jamais imposer) des mots clés. Pour ce faire, l'enseignant doit se soumettre à l'exercice et être en mesure de décrire, pas à pas, sa propre démarche. En bref, la pédagogie par l'exemple préconisée par D. Seleskovitch.

L'objectif de notre enseignement consistant à conduire l'apprenti-interprète sur la voie des idées et non des mots, il faut le faire s'affronter à des énoncés "dont le sens ne pourra être appréhendé que par le biais d'un raisonnement contextuel ... un raisonnement analogique par recoupements conceptuels 13 ", En guise d'exemple, soumettre aux étudiants des proverbes (en langue maternelle) parmi les plus connus et les amener à en extraire une seule notion clé constitue un excellent entraînement qui, bien que difficile, dans la plupart des cas suscite une prise de conscience sans équivoque de l'objectif recherché.

\section{DE L'INTERPRÉTATION UNILINGUE}

Enseigner un savoir-faire nécessite à la fois une pédagogie par l'exemple, une explication des principes qui régissent ce savoir-faire, et la communication d'un certain nombre de recettes ou ficelles du métier - ceci pour l'aspect positif de l'enseignement ; pour l'aspect correctif, la pédagogie qui s'attaque aux erreurs, il s'agit non seulement de les redresser individuellement, mais d'en indiquer les causes pour ne pas traiter les symptômes mais guérir la maladie14. (Danica Seleskovitch)

Nous ne reviendrons pas sur l'intérêt pédagogique des exercices d'interprétation unilingue en début d'apprentissage clairement exposé par D. Gile : "L'exercice unilingue favorise ... la focalisation des efforts sur les problèmes fondamentaux en éliminant d'emblée toutes les difficultés pratiques liées au bilinguisme, qui occultent souvent la véritable nature de l'interprétation...15

Les exemples pédagogiques sont légion et les textes qui peuvent faire l'objet de tels exercices foisonnent. Nous en décrirons brièvement un. Le texte qui suit, extrait de l'allocution d'ouverture de $\mathbf{M}$. Monory aux Rencontres internationales du ministère de l'Économie ${ }^{16}$, soumis à plusieurs reprises à divers groupes d'étudiants, a engendré les mêmes problèmes : d'une part, la non prise en compte par les 
étudiants des éléments prosodiques-source les empêche de suivre le raisonnement, les arguments, les enchaînements ; d'autre part, leur incapacité d'évoquer mentalement une situation ${ }^{17}$, des images, les empêche d'appréhender le vouloir-dire de l'orateur.

La société industrielle connaît des changements profonds; de nouveaux producteurs apparaissent sur la scène mondiale ; l'évolution technologique bouleverse les conditions de la production et du travail.

- Les nouveaux producteurs sont-ils à terme, pour les économies industrielles, une chance ou une menace? - Leur apparition est un fait important. Mais j'ajouterai que, vis-à-vis de la plupart des pays d'exportation de produits à bon marché (comme la Corée du Sud par exemple), la balance commerciale d'un pays comme la France est régulièrement excédentaire.

- L'évolution technologique, la diffusion de l'électronique, de l'informatique, de la bureautique bouleversent déjà la structure des économies industrielles. Ces bouleversements iront en s'accélérant.

Nous parlions, il y a quelques années, du choc du futur : nous y sommes.

Déjà au Japon, fonctionnement des aciéries automatisées en quasi-totalité et demain, sans doute, d'autres grands secteurs comme l'automobile s'engageront-ils dans la voie de la production automatique.

Face à ce texte, dont la production orale ne dure guère plus d'une minute, l'interprète doit pouvoir dégager une ossature sinon simple du moins classique : une affirmation générale (la transformation de la société industrielle) justifiée par deux constatations (l'apparition de nouveaux producteurs et les bouleversements dus aux nouvelles technologies) commentées séparément ; une conclusion (choc du futur) illustrée d'une autre constatation (l'exemple du Japon). Or, rares ont été les étudiants (même lorsque l'exercice s'est réalisé avec prise de notes) capables de faire apparaître clairement cette ossature dans leur restitution. Causes : l'étudiant ne prend pas en compte les deux pauses de l'orateur précédant "de nouveaux producteurs apparaissent... " et "l'évolution technologique bouleverse..." ni, plus loin, les intonations de l'orateur lorsqu'il reprend ces deux constatations pour les commenter. Seules la conclusion et son illustration sont clairement perçues (notons cependant que plusieurs étudiants ne voient pas l'allusion contenue dans "le choc du futur $\%$.

Les difficultés de compréhension du contenu proprement dit se concentrent dans le commentaire concernant les nouveaux producteurs. Après audition du texte, rares sont les étudiants capables de donner une réponse satisfaisante à la question de l'orateur. Certains vont même jusqu'à affirmer que la question reste sans réponse...

Causes :

- l'étudiant ne se pose pas la question de savoir qui sont ces nouveaux producteurs la première fois qu'il entend l'expression. Cette anticipation (qui ne requiert pourtant pas de connaissances particulières) lui aurait permis de capter le sens de la question : Ces pays représentent-ils un danger pour nous?

- l'étudiant n'assume pas la position de l'orateur. Il ne se met pas " dans la peau " de l'orateur et, de ce fait, ne "sent " pas le danger, la menace. Il ne s'efforce pas non plus de " voir " mentalement tous ces produits "Made in Hong Kong " qui déferlent sur tous les marchés.

- l'étudiant néglige l'importance de certains éléments formels tels que " mais " (dont le rôle est capital), "régulièrement ", l'intonation accompagnant "Leur apparition est un fait important".

Finalement, bon nombre d'erreurs proviennent du fait que l'étudiant ne s'efforce pas, comme le dit Christopher Thiéry, de « juger l'orateur tout en l'écoutant, tant sur la manière dont il exprime ses idées que sur les idées elles-mêmes ${ }^{18} \%$. Thiéry poursuit : "L'étudiant sera ainsi amené progressivement à concilier deux attitudes en apparence contradictoires. Il lui faut d'abord une certaine distanciation visà-vis de l'orateur, qui lui permettra de répondre à tout moment à ces deux questions : "quelle est sa thèse ? (est-il pour ou contre? " et : " est-ce qu'il la défend bien? " Mais en même temps il ne doit pas hésiter à s'engager lui-même, quitte à juger sévèrement celui dont il est néanmoins le porte-parole fidèle. »

L'interprétation unilingue, on le voit, touche au cœur même du savoir-faire défini plus haut par Jean Delisle. Il n'est donc pas inutile d'établir une liste systématique des difficultés qui lui sont propres en vue de mettre au point une série d'exercices ponctuels. On trouve dans un travail du Groupe de réflexion et de recherche sur la pédagogie de l'interprétation de Mons ${ }^{19}$ un relevé précis sur des problèmes rencontrés par l'interprète unilingue. Au niveau du décodage, les difficultés sont d'ordre sémantique et lexicologique (compréhension du message), informationnel ou culturel (appréhension du contenu de ce message) et logique ou intellectuel (son entendement). Au niveau de l'encodage, les problèmes sont techniques (voix, articulation, diction), linguistiques (morphosyntaxiques, lexicaux, stylistiques) et psychologiques (présentation, contact, confiance en soi, fiabilité).

L'exemple donné plus haut se réfère au niveau du décodage et peut faire l'objet d'exercices d'interprétation, d'extraction des notions clés, de prise de notes, etc. Il va de soi que, avec la reformulation, l'exercice devient complet et permet l'observation des aptitudes d'encodage des étudiants et leur correction éventuelle. II convient dès lors que l'ensei- 
gnant soit extrêmement attentif aux erreurs ou déficiences possibles tant au niveau du décodage que de l'encodage. Nous recommandons que toute correction s'effectue en deux parties : d'abord les fautes et trouvailles de forme, ensuite les erreurs et réussites de contenu. Étant donné le caractère global inéluctable de tout exercice d'interprétation, l'enseignant veillera aussi à souligner l'incidence relative de tel défaut sur l'ensemble de la performance.

\section{DE L'INTERPRÉTATION BILINGUE}

Le transfert d'une langue à une autre, la véritable interprétation, ne modifie en rien les commentaires énoncés ci-dessus mais entraîne des difficultés supplémentaires inhérentes au transfert linguistique ${ }^{20}$.

Sortant quelque peu du cadre de l'initiation à l'interprétation, nous nous bornerons à énumérer les clifficultés qui viennent s'ajouter à l'exercice unilingue lorsque l'étudiant se voit confronté à deux systèmes linguistiques différents. Nous reprendrons la classification présentée par le Groupe de réflexion de Mons ${ }^{21}$ en l'illustrant d'exemples recueillis au cours de l'interprétation vers l'espagnol du texte de $\mathbf{M}$. Monory.

En ce qui concerne le décodage, le Groupe mentionne quatre difficultés qui relèvent de la compréhension :

- linguistique :

- lexique : bouleverser, à terme, ...

- morphosyntaxe : iront en s'accélérant, sans doute ... s'engageront-ils, ...

- stylistique : j'ajouterai, nous parlions, nous y sommes, ...

- périlinguistique : bureautique, aciéries, pays d'exportation de produits à bon marché, ...

- extralinguistique : nouveaux producteurs, choc du futur, ..

-_ intellectuelle : les difficultés linguistiques, périlin guistiques et extralinguistiques que présente la phrase "Mais j'ajouterai que ... excédentaire" masquent l'idée et en empêchent la véritable compréhension.

Quant à l'encodage ou reformulation, «le transfert linguistique suscite des calques lexicaux et syntaxiques, des erreurs morphosyntaxiques, des lourdeurs stylistiques qui entachent la langue d'arrivée 22 \%. Dans l'interprétation espagnole de notre texte, on peut s'attendre aux calques suivants : "escena" au lieu de "escenario" pour "scène", "sin duda" au lieu de "tal vez" ou "probablemente" pour "sans doute", "sector automóvil " au lieu de "sector automotor" pour "l'automobile", etc.

\section{CONCLUSION}

Des trois questions posées dans l'introdution, seule la troisième a obtenu une réponse : apprendre à nos étudiants à communiquer, développer en eux le potentiel d'écoute, de pensée et d'expression, les inciter à accroître et diversifier leur savoir, leur faire prendre conscience des divers systèmes sociocultu- rels et leur inculquer les meilleures attitudes. La réponse aux deux premières questions se trouve dans la capacité de l'étudiant-de-la-rue à tirer parti de cet entraînement. Les efforts à produire seront différents pour chacun et la somme de travail individuel constituera bien souvent la clé du succès.

Les aptitudes requises pour l'exercice de notre profession ne sont pas un don du ciel. Elles exigent une préparation méthodique et un entraînement systématique au terme desquels on constate toutefois qu'il y a beaucoup d'appelés ... mais peu d'élus. Que l'on ne me fasse pas dire ce que je n'ai pas dit !

Comprendre un contenu et le rendre intelligible est utile à chacun, mais pour un traducteur [interprète] c'est vital. (Michèle GhenetHottois ${ }^{23}$ )

GEORGES L. BASTIN

École de langues modernes, Université centrale du Venezuela, Venezuela

Notes

1. Jean Delisle (1984) : "Plaidoyer en faveur du renouveau de l'enseignement de la traduction professionnelle ", in Cahiers de traductologie, $\mathrm{n}^{\circ}$ 5, Éd. de l'Université d'Ottawa, p. 293

2. L'École de langues modernes de l'Université centrale du Venezuela prévoit, pour la licence de traduction-interprétation, un cours d'initiation à l'interprétation en langue maternelle d'une durée de deux semestres. Les études s'étalent sur dix semestres dont six sont consacrés à la traduction et quatre à l'interprétation consécutive et simultanée. Aucun examen d'admission n'est requis.

3. Daniel Gile (1983) : "L'enseignement de l'interprétation : utilisation des exercices unilingues en début d'apprentissage " in Traduire, $\mathrm{n}^{\circ} 113$, janvier, $\mathrm{p} .7$.

4. Roda Roberts (1981) : " Translation : An Act of Communication" in Bulletin of the CAAL, Autumn, pp. 151-163. (1984) : " Translation and Communication ", communication présentée au colloque " La traduction dans le processus de la communication ", UCV, Caracas, juillet.

5. David K. Berlo (1975) : El proceso de la comunicación, Editorial El Ateneo, Buenos Aires.

6. Danica Seleskovitch (1975) : Langage, langues et mémoire, Paris, Lettres modernes, Minard.

7. In Traduire, $\mathrm{n}^{\mathrm{O}} 120$, juillet $1984, \mathrm{p} .4$.

8. Christopher Thiery (1981): "L'enseignement de la prise de notes en interprétation consécutive : un faux problème? " in Cahiers de traductologie, $n^{\circ} 4$, Ed. de l'Université d'Ottawa, Canada, p. 101.

9. Danica Seleskovitch, op. cit., pp. 7-8.

10. Georges L. Bastin (1983) : "Pour une méthodologie de la traduction" in Revue de phonétique appliquée, nos 66-67-68, p. 214. 
11. Jean Delisle (1980) : "l'Analyse du discours comme méthode de traduction", Cahiers de traductologie, $\mathbf{n}^{\circ}$ 2, Éd. de l'Université d'Ottawa, Canada, p. 146.

12. David K. Berlo, op. cit., p. 36.

13. Georges L. Bastin, op. cit., p. 217.

14. Danica Seleskovitch (1983): "Enseignement de la traduction à vue" in Revue de phonétique appliquée, $\mathrm{n}^{\mathrm{os}}$ 66-67-68, p. 166.

15. Daniel Gile, op. cit., p. 7.

16. UNESCO (1981) : les Années 80. Faits et choix économiques, PUF, p. 9.

17. Danica Seleskovitch (1975) : Langage, langues et mémoire, Paris, Lettres modernes, Minard, p. 58

18. Christopher Thiery, op. cit., p. 108.

19. "Collectif I": groupe de réflexion et de recherche sur la pédagogie de l'interprétation, "Pédagogie de l'interprétation : projets de recherches " in Vingt ans d'enseignement et de recherche en traduction et en interprétation de conférence, Université de Mons, École d'interprètes internationaux, 1983, pp. 129-132.

20. "Collectif I", op. cit., p. 131.

21. "Collectif I", op. cit., p. 131.

22. "Collectif I", op. cit., p. 132.

23. Michèle Ghenet-Hottois (1983) : "Comprendre et se faire comprendre" in Vingt ans d'enseignement et de recherche en traduction et en interprétation de conférence, Université de Mons, Ecole d'interprètes internationaux, p. 23. 